PROCEEDINGS OF THE

AMERICAN MATHEMATICAL SOCIETY

Volume 131, Number 6, Pages 1803-1812

S 0002-9939(02)06727-8

Article electronically published on October 2, 2002

\title{
ON SAMPLING THEORY ASSOCIATED WITH THE RESOLVENTS OF SINGULAR STURM-LIOUVILLE PROBLEMS
}

\author{
M. H. ANNABY \\ (Communicated by Carmen C. Chicone)
}

\begin{abstract}
This paper is concerned with the sampling theory associated with resolvents of eigenvalue problems. We introduce sampling representations for integral transforms whose kernels are Green's functions of singular SturmLiouville problems provided that the singular points are in the limit-circle situation, extending the results obtained in the regular problems.
\end{abstract}

\section{INTRODUCTION}

In their article, 14, Haddad et al. indicated that the celebrated WhittakerKotel'nikov-Shannon sampling theorem can be derived using the eigenfunctions expansion of the Green's function of first order eigenvalue problems. They also gave examples including second order regular Sturm-Liouville systems; cf. [23. The work is extended to $n$th order regular operators, which are either self-adjoint or non-self-adjoint in [2, 22]. Thus integral transforms whose kernels are the Green's function of the eigenvalue problems are reconstructed from their values at the eigenvalues of the associated problems via (interpolation) sampling series. As an example (cf. [2]), consider the problem

$$
\begin{gathered}
-y^{\prime \prime}=\lambda y, \quad 0 \leq x \leq \pi, \quad \lambda \in \mathbb{C}, \\
y(0)+y(\pi)=0, \quad y^{\prime}(0)+y^{\prime}(\pi)=0 .
\end{gathered}
$$

This problem has the double eigenvalues $\lambda_{n}=(2 n-1)^{2}, n=1,2, \ldots$, with the corresponding eigenfunctions $\varphi_{n}^{1}(x)=\sin (2 n-1) x, \varphi_{n}^{2}(x)=\cos (2 n-1) x$. The Green's function of this problem is

$$
G(x, \xi, \lambda)=\frac{1}{4(1+\cos \sqrt{\lambda} \pi)}\left\{\begin{array}{cc}
2 \sin \sqrt{\lambda} \pi \cos (\xi-x) \\
-(1+\cos \sqrt{\lambda} \pi) \sin (\xi-x), \quad x \geq \xi, \\
2 \sin \sqrt{\lambda} \pi \cos (x-\xi) \\
-(1+\cos \sqrt{\lambda} \pi) \sin (x-\xi), \quad x \leq \xi .
\end{array}\right.
$$

Received by the editors November 15, 2000 and, in revised form, January 18, 2002.

2000 Mathematics Subject Classification. Primary 41A05, 34B05, 94A20.

Key words and phrases. Sampling theory, singular Sturm-Liouville problems, Green's function, resolvent kernels, Legendre and Bessel functions. 
Let $\xi_{0} \in[0, \pi]$ be a fixed point and $\Psi(x, \lambda):=p(\lambda) G\left(x, \xi_{0}, \lambda\right), \lambda \in \mathbb{C}, p(\lambda):=$ $\prod_{n=1}^{\infty}\left(1-\frac{\lambda}{(2 n-1)^{2}}\right)$. The function $\Psi(x, \lambda)$ is an entire function of $\lambda$ for every $x$ since the simple poles of $G\left(x, \xi_{0}, \lambda\right)$ are exactly the simple zeros of the infinite product. Knowing that $p(\lambda)=\cos \sqrt{\lambda} \pi / 2$, the integral transform

$$
f(\lambda)=\int_{0}^{\pi} g(x) \Psi(x, \lambda) d x, \quad g(\cdot) \in L^{2}(0, \pi)
$$

has the sampling representation

$$
f(\lambda)=\sum_{n=1}^{\infty}(-1)^{n} f\left(4 n^{2}-4 n+1\right) \frac{4(2 n-1) \cos \sqrt{\lambda} \pi / 2}{\pi\left(\lambda-4 n^{2}+4 n-1\right)} .
$$

Series (1.5) converges absolutely and uniformly on compact subsets of the complex plane. For details, see [2], and for other articles dealing with sampling theory associated with Green's functions, see, e.g., 3]. We would like to mention that Green's function of eigenvalue problems is the resolvent kernel associated with these problems.

The aim of this article is to investigate the situation when regular problems are replaced by singular ones. We prove that integral transforms whose kernels are Green's functions of singular Sturm-Liouville problems can be recovered from their values at the eigenvalues. In [4, 9, 20, 21], the study of Kramer's sampling theorem, [5, 15], associated with singular problems was carried out where the kernels of the sampled transforms are solutions of the problems. In the next section we introduce the singular Sturm-Liouville problem as well as the associated Green's function. We assume that the limit-circle case holds at any singular point. Then, boundary conditions at the singular points are defined using the approach established by Fulton in 11, [12]. Then we state the main result of the present paper. Section 3 contains a proof of the main result introduced in $\S 2$. The last section, $\S 4$, is devoted to applications including Legendre and Bessel functions.

\section{The MAIN RESUlT}

Consider the Sturm-Liouville equation

$$
\ell(y):=-y^{\prime \prime}+q(x) y=\lambda y, \quad-\infty \leq a \leq x<\infty, \lambda \in \mathbb{C},
$$

and the boundary condition

$$
\cos \alpha y(a)+\sin \alpha y^{\prime}(a)=0
$$

where $q(\cdot)$ is continuous on $[a, \infty)$. Moreover, the limit-circle case is assumed to be satisfied at $\infty$. Thus, we must add another condition at $\infty$ to define an eigenvalue problem. Following the theory derived by Fulton in [11, 12, we may define the following boundary condition at $\infty$ :

$$
\cos \gamma(S y)_{1}(\infty)+\sin \gamma(S y)_{2}(\infty)=0
$$

where

$$
(S y)_{1}(\infty)=\lim _{x \rightarrow \infty} W_{x}(y, v), \quad(S y)_{2}(\infty)=\lim _{x \rightarrow \infty} W_{x}(y, u)
$$

and $u, v$ are two solutions of $(2.1)$ when $\lambda=0$ satisfying $W_{x}(u, v) \equiv 1$ on $[a, \infty)$. Here $W_{x}(u, v)$ denotes the Wronskian of $u, v$, i.e., $W_{x}(u, v):=u(x) v^{\prime}(x)-u^{\prime}(x) v(x)$. Consequently, our problem is the eigenvalue problem (2.1)-(2.3) which we denote by $(*)$. According to the limit-circle situation [19], the spectrum of $\left(^{*}\right)$ is discrete, 
simple and can be ordered in a sequence of real numbers $\left\{\lambda_{n}\right\}_{n=0}^{\infty}$ with no finite limit points. Moreover, all solutions of $(2.1)$ are $L^{2}(a, \infty)$-functions; cf. [18], [19].

Let $\phi(\cdot, \lambda)$ and $\chi(\cdot, \lambda)$ be the solutions of $(2.1)$ defined by the initial conditions

$$
\begin{gathered}
\phi(a, \lambda)=\sin \alpha, \quad \phi^{\prime}(a, \lambda)=-\cos \alpha, \\
(S \chi(\cdot, \lambda))_{1}(\infty)=\sin \gamma, \quad(S \chi(\cdot, \lambda))_{2}(\infty)=-\cos \gamma, \quad \lambda \in \mathbb{C} .
\end{gathered}
$$

These solutions are entire functions of $\lambda$ for every $x$. We denote their Wronskian, which is independent of $x$, by $\omega_{\alpha, \gamma}(\lambda)$, i.e.,

$$
\omega_{\alpha, \gamma}(\lambda):=W_{x}(\phi(\cdot, \lambda), \chi(\cdot, \lambda))
$$

The function $\omega_{\alpha, \gamma}(\lambda)$ is entire in $\lambda$ with (simple) zeros that are exactly the eigenvalues of $(*)$, [12]. Every eigenvalue $\lambda_{n}$ has one and only one linearly independent eigenfunction, which might be either $\phi\left(\cdot, \lambda_{n}\right)$ or $\chi\left(\cdot, \lambda_{n}\right)$. The sequence of eigenfunctions corresponding to the sequence of eigenvalues $\left\{\lambda_{n}\right\}_{n=0}^{\infty}$ is either $\left\{\phi\left(\cdot, \lambda_{n}\right)\right\}_{n=0}^{\infty}$ or $\left\{\chi\left(\cdot, \lambda_{n}\right)\right\}_{n=0}^{\infty}$. Each sequence is an orthogonal basis of $L^{2}(a, \infty)$ and (cf. [12]) there are constants $\kappa_{n}$ such that

$$
\chi\left(x, \lambda_{n}\right)=\kappa_{n} \phi\left(x, \lambda_{n}\right), \quad \kappa_{n} \in \mathbb{R}-\{0\} .
$$

If $\lambda \in \mathbb{C}$ is not an eigenvalue, then the Green's function of problem $\left(^{*}\right)$ is given by $(12])$

$$
G(x, \xi, \lambda)=\frac{1}{\omega_{\alpha, \gamma}(\lambda)} \begin{cases}\phi(\xi, \lambda) \chi(x, \lambda), & a \leq \xi \leq x<\infty, \\ \phi(x, \lambda) \chi(\xi, \lambda), & a \leq x \leq \xi<\infty .\end{cases}
$$

The Green's function (2.9) is a meromorphic function of $\lambda$ with simple poles at the eigenvalues. $\kappa_{n} \phi\left(x, \lambda_{n}\right) \phi\left(\xi, \lambda_{n}\right) / \omega_{\alpha, \gamma}^{\prime}\left(\lambda_{n}\right)$ is the residue of $G(x, \xi, \lambda)$ at an eigenvalue $\lambda_{n}$ [12]. Since $\phi(\cdot, \lambda)$ and $\chi(\cdot, \lambda)$ are $L^{2}(a, \infty)$-functions, then $G(x, \xi, \lambda)$ is also in $L^{2}([a, \infty) \times[a, \infty))$ when $\lambda$ is not an eigenvalue. Moreover, it is not hard to see that

$$
\begin{aligned}
\int_{a}^{\infty} & \int_{a}^{\infty}|G(x, \xi, \lambda)|^{2} d x d \xi \\
& \leq \frac{2}{\left|\omega_{\alpha, \gamma}(\lambda)\right|^{2}}\|\phi(\cdot, \lambda)\|^{2}\|\chi(\cdot, \lambda)\|^{2}<\infty, \quad \lambda \in \mathbb{C}-\left\{\lambda_{n}\right\}_{n=0}^{\infty} .
\end{aligned}
$$

Let $\xi_{0} \in[a, \infty)$ be an arbitrary fixed point. Define the function $\Phi(\cdot, \lambda)$ to be

$$
\Phi(x, \lambda):=\omega_{\alpha, \gamma}(\lambda) G\left(x, \xi_{0}, \lambda\right), \quad \lambda \in \mathbb{C}, a \leq x<\infty .
$$

Obviously, $\Phi(x, \lambda)$ is an entire function of $\lambda$ for all $x$ and it belongs to $L^{2}(a, \infty)$ for any $\lambda \in \mathbb{C}$. Before we state the main result of the paper, we mention a couple of things. The first one is Green's identity, which is a needed tool to prove the sampling theorem of this article. Green's identity states that for suitable functions $y, z$ and $a \leq x_{1} \leq x_{2}<\infty$, we have

$$
\int_{x_{1}}^{x_{2}} z(x) \ell(y(x))-y(x) \ell(z(x)) d x=\left.W_{x}(y, z)\right|_{x_{1}} ^{x_{2}} .
$$

See [7, p. 86] for a more general form. The second thing we would like to mention is that although the main result is derived in the case when we have a half-line interval, the results might be extended when the left end point is also singular and the right end point is singular or regular. In case we have a finite singular point, 
this means that $q(\cdot)$ has a singularity at this point. Also the expression $\ell(\cdot)$ may be replaced by $-\left(p(x) y^{\prime}\right)^{\prime}+q(x) y=\lambda r(x) y, r, 1 / p, q \in L_{\mathrm{loc}}^{1}[a, \infty), r(x)$ is positive and $p(x)$ does not vanish at any point of $[a, \infty)$. See [9, 10, 12].

Theorem 2.1. Let $\Phi(\cdot, \lambda)$ be the function defined in (2.11) and let $g(\cdot) \in L^{2}(a, \infty)$. Define the integral transform

$$
f(\lambda)=\int_{a}^{\infty} \bar{g}(x) \Phi(x, \lambda) d x
$$

Then $f(\lambda)$ is an entire function of $\lambda$ that admits the sampling expansion

$$
f(\lambda)=\sum_{n=0}^{\infty} f\left(\lambda_{n}\right) \frac{\omega_{\alpha, \gamma}(\lambda)}{\left(\lambda-\lambda_{n}\right) \omega_{\alpha, \gamma}^{\prime}\left(\lambda_{n}\right)} .
$$

Moreover, expansion (2.14) converges absolutely and uniformly on compact subsets of the complex plane.

\section{A PRoOf OF the MAIN RESUlT}

In the following we give a proof of Theorem 2.1 above. Let $\mathbb{N}_{0}$ denote the set of non-negative integers and $\varphi_{n}(x):=\phi\left(x, \lambda_{n}\right), n \in \mathbb{N}_{0}$. The eigenfunctions can be chosen to be real-valued. We start with the following lemma.

Lemma 3.1. Let $\Omega$ be a compact subset of $\mathbb{C}$. Then, there exists a positive constant $C(\Omega)$, which does not depend on $\lambda$, such that

$$
\|\Phi(\cdot, \lambda)\| \leq C(\Omega), \quad \text { for all } \lambda \in \Omega \text {. }
$$

Proof. Since $\Omega$ is compact, then from [7, Theorem 2.1, p. 225], there is a positive constant $\delta_{1}$, which is independent of $\lambda$, such that

$$
\|\phi(\cdot, \lambda)\|,\|\chi(\cdot, \lambda)\| \leq \delta_{1} \quad \text { for all } \lambda \in \Omega .
$$

Also, the compactness of $\Omega$ and the fact that both $\phi\left(\xi_{0}, \lambda\right)$ and $\chi\left(\xi_{0}, \lambda\right)$ are entire imply that there is $\delta_{2}>0$, which is independent of $\lambda$, such that

$$
\left|\phi\left(\xi_{0}, \lambda\right)\right|^{2},\left|\chi\left(\xi_{0}, \lambda\right)\right|^{2}<\delta_{2} \quad \text { for all } \lambda \in \Omega \text {. }
$$

Choosing $C(\Omega)=\sqrt{2 \delta_{1} \delta_{2}}$, the lemma is proved

Proof of Theorem 2.1. We assume first that $\varphi_{n}\left(\xi_{0}\right) \neq 0$ for all $n \in \mathbb{N}_{0}$. Since the functions $g(\cdot)$ and $\Phi(\cdot, \lambda)$ belong to $L^{2}(a, \infty)$ for all $\lambda$ and since the sequence $\left\{\varphi_{n}(\cdot)\right.$ : $\left.n \in \mathbb{N}_{0}\right\}$ is an orthogonal basis of $L^{2}(a, \infty)$, then applying Parseval's equality to the integral transform (2.13), we obtain

$$
f(\lambda)=\sum_{n=0}^{\infty} \frac{\bar{g}(n) \widehat{\Phi}(n, \lambda)}{\left\|\varphi_{n}(\cdot)\right\|^{2}}, \quad \lambda \in \mathbb{C},
$$

where

$$
\widehat{g}(n)=\int_{a}^{\infty} g(x) \varphi_{n}(x) d x, \quad \widehat{\Phi}(n, \lambda)=\int_{a}^{\infty} \Phi(x, \lambda) \varphi_{n}(x) d x
$$

are the Fourier coefficients of $g(\cdot)$ and $\Phi(\cdot, \lambda)$ respectively. Let $\lambda \in \mathbb{C}$ and $n \in \mathbb{N}_{0}$ be fixed. Then for every $\lambda \in \mathbb{C}-\left\{\lambda_{n}\right\}_{n=0}^{\infty}$ we have

$$
\widehat{\Phi}(n, \lambda)=\lim _{M \rightarrow \infty} \int_{a}^{M} \Phi(x, \lambda) \varphi_{n}(x) d x .
$$


Using the definition of $\Phi(\cdot, \lambda)$,

$$
\begin{aligned}
\int_{a}^{M} \Phi(x, \lambda) \varphi_{n}(x) d x= & \chi\left(\xi_{0}, \lambda\right) \int_{a}^{\xi_{0}} \phi(x, \lambda) \varphi_{n}(x) d x \\
& +\phi\left(\xi_{0}, \lambda\right) \int_{\xi_{0}}^{M} \chi(x, \lambda) \varphi_{n}(x) d x,
\end{aligned}
$$

where $M$ is sufficiently large. Now we use Green's identity (2.12) and the fact that $\phi(\cdot, \lambda)$ and $\chi(\cdot, \lambda)$ are solutions of $(2.1)$ to obtain

$$
\begin{aligned}
\left(\lambda-\lambda_{n}\right) \int_{a}^{M} \Phi(x, \lambda) \varphi_{n}(x) d x & =\chi\left(\xi_{0}, \lambda\right)\left[\phi\left(\xi_{0}, \lambda\right) \varphi_{n}^{\prime}\left(\xi_{0}\right)-\phi^{\prime}\left(\xi_{0}, \lambda\right) \varphi_{n}\left(\xi_{0}\right)\right. \\
& \left.-\phi(a, \lambda) \varphi_{n}^{\prime}(a)+\phi^{\prime}(a, \lambda) \varphi_{n}(a)\right] \\
& +\phi\left(\xi_{0}, \lambda\right)\left[\chi(M, \lambda) \varphi_{n}^{\prime}(M)-\chi^{\prime}(M, \lambda) \varphi_{n}(M)\right. \\
& \left.-\chi\left(\xi_{0}, \lambda\right) \varphi_{n}^{\prime}\left(\xi_{0}\right)+\chi^{\prime}\left(\xi_{0}, \lambda\right) \varphi_{n}\left(\xi_{0}\right)\right] .
\end{aligned}
$$

Simplifying (3.5), we obtain

$$
\begin{aligned}
\left(\lambda-\lambda_{n}\right) \int_{a}^{M} \Phi(x, \lambda) \varphi_{n}(x) d x & =\varphi_{n}\left(\xi_{0}\right)\left[\phi\left(\xi_{0}, \lambda\right) \chi^{\prime}\left(\xi_{0}, \lambda\right)-\phi^{\prime}\left(\xi_{0}, \lambda\right) \chi\left(\xi_{0}, \lambda\right)\right] \\
& +\chi\left(\xi_{0}, \lambda\right)\left[\varphi_{n}(a) \phi^{\prime}(a, \lambda)-\varphi_{n}^{\prime}(a) \phi(a, \lambda)\right] \\
& +\phi\left(\xi_{0}, \lambda\right)\left[\varphi_{n}^{\prime}(M) \chi(M, \lambda)-\varphi_{n}(M) \chi^{\prime}(M, \lambda)\right] .
\end{aligned}
$$

From (2.5) and (2.7), one gets

$$
\left(\lambda-\lambda_{n}\right) \int_{a}^{M} \Phi(x, \lambda) \varphi_{n}(x) d x=\varphi_{n}\left(\xi_{0}\right) \omega_{\alpha, \gamma}(\lambda)+\phi\left(\xi_{0}, \lambda\right) W_{M}\left(\chi(\cdot, \lambda), \varphi_{n}(\cdot)\right) .
$$

From [12] p. 55],

$$
W_{M}\left(\chi(\cdot, \lambda), \varphi_{n}(\cdot)\right)=D_{M}\left(S\left(\chi(\cdot, \lambda), \varphi_{n}(\cdot)\right)\right):=\left|\begin{array}{ll}
(S \chi(\cdot, \lambda))_{1}(M) & \left(S \varphi_{n}(\cdot)\right)_{1}(M) \\
(S \chi(\cdot, \lambda))_{2}(M) & \left(S \varphi_{n}(\cdot)\right)_{2}(M)
\end{array}\right| .
$$

Taking the limit in (3.8) when $M \longrightarrow \infty$, we obtain

$$
W_{\infty}\left(\chi(\cdot, \lambda), \varphi_{n}(\cdot)\right)=(S \chi(\cdot, \lambda))_{1}(\infty)\left(S \varphi_{n}(\cdot)\right)_{2}(\infty)-(S \chi(\cdot, \lambda))_{2}(\infty)\left(S \varphi_{n}(\cdot)\right)_{1}(\infty) .
$$

From (2.6) and since $\varphi_{n}(\cdot)$ is an eigenfunction, $W_{\infty}\left(\chi(\cdot, \lambda), \varphi_{n}(\cdot)\right)=0$. Therefore, if we take the limit in (3.7) as $M \longrightarrow \infty$, we get

$$
\int_{a}^{\infty} \Phi(x, \lambda) \varphi_{n}(x) d x=\varphi_{n}\left(\xi_{0}\right) \frac{\omega_{\alpha, \gamma}(\lambda)}{\lambda-\lambda_{n}} .
$$

If $\lambda$ approaches $\lambda_{n}$ in the previous equation, then

$$
\int_{a}^{\infty} \Phi\left(x, \lambda_{n}\right) \varphi_{n}(x) d x=\varphi_{n}\left(\xi_{0}\right) \omega_{\alpha, \gamma}^{\prime}\left(\lambda_{n}\right)
$$

Relation (2.8) and the definition of $\Phi(\cdot, \lambda)$ imply that

$$
\int_{a}^{\infty} \Phi\left(x, \lambda_{n}\right) \varphi_{n}(x) d x=\kappa_{n} \varphi_{n}\left(\xi_{0}\right)\left\|\varphi_{n}(\cdot)\right\|^{2} .
$$


Hence $\left\|\varphi_{n}(\cdot)\right\|^{2}=\kappa_{n}^{-1} \omega_{\alpha, \gamma}^{\prime}\left(\lambda_{n}\right)$. Substituting from (3.10) and (3.12) in (3.2) we have

$$
f(\lambda)=\sum_{n=0}^{\infty} \bar{g}(n) \frac{\kappa_{n} \varphi_{n}\left(\xi_{0}\right) \omega_{\alpha, \gamma}(\lambda)}{\left(\lambda-\lambda_{n}\right) \omega_{\alpha, \gamma}^{\prime}\left(\lambda_{n}\right)} .
$$

Moreover, for any $n \in \mathbb{N}_{0}$ we can see that

$$
f\left(\lambda_{n}\right)=\int_{a}^{\infty} \bar{g}(x) \Phi\left(x, \lambda_{n}\right) d x=\kappa_{n} \overline{\widehat{g}}(n) \varphi_{n}\left(\xi_{0}\right) .
$$

Expansion (2.14) results by combining (3.13) and (3.14) and the convergence is pointwise on $\mathbb{C}$. To prove absolute convergence on $\mathbb{C}$, let $\lambda \in \mathbb{C}$ be arbitrary. Then

$$
\begin{aligned}
\sum_{n=0}^{\infty}\left|f\left(\lambda_{n}\right) \frac{\omega_{\alpha, \gamma}(\lambda)}{\left(\lambda-\lambda_{n}\right) \omega_{\alpha, \gamma}^{\prime}\left(\lambda_{n}\right)}\right| & =\sum_{n=0}^{\infty}\left|\widehat{g}(n) \frac{\widehat{\Phi}(n, \lambda)}{\left\|\varphi_{n}(\cdot)\right\|^{2}}\right| \\
& \leq\left(\sum_{n=0}^{\infty}\left|\frac{\widehat{g}(n)}{\left\|\varphi_{n}(\cdot)\right\|}\right|^{2}\right)^{\frac{1}{2}}\left(\sum_{n=0}^{\infty}\left|\frac{\widehat{\Phi}(n, \lambda)}{\left\|\varphi_{n}(\cdot)\right\|}\right|^{2}\right)^{\frac{1}{2}}
\end{aligned}
$$

The right-hand side of the last equation is $\|g(\cdot)\|\|\Phi(\cdot, \lambda)\|<\infty$, implying absolute convergence. As for uniform convergence, let $\Omega \subset \mathbb{C}$ be compact. Then from Lemma 3.1 above, there exists a positive constant $C(\Omega)$, which is independent of $\lambda$ such that $\|\Phi(\cdot, \lambda)\| \leq C(\Omega)$, for all $\lambda \in \Omega$. Again, using the Cauchy-Schwarz inequality we have for any positive integer $N$,

$$
\begin{aligned}
\left|f(\lambda)-\sum_{n=0}^{N-1} f\left(\lambda_{n}\right) \frac{\omega_{\alpha, \gamma}(\lambda)}{\left(\lambda-\lambda_{n}\right) \omega_{\alpha, \gamma}^{\prime}\left(\lambda_{n}\right)}\right| & \leq \sum_{n=N}^{\infty}\left|\widehat{g}(n) \frac{\widehat{\Phi}(n, \lambda)}{\left\|\varphi_{n}(\cdot)\right\|^{2}}\right| \\
& \leq\left(\sum_{n=N}^{\infty}\left|\frac{\widehat{g}(n)}{\left\|\varphi_{n}(\cdot)\right\|}\right|^{2}\right)^{\frac{1}{2}}\left(\sum_{n=N}^{\infty}\left|\frac{\widehat{\Phi}(n, \lambda)}{\left\|\varphi_{n}(\cdot)\right\|}\right|^{2}\right)^{\frac{1}{2}} \\
& \leq C(\Omega)\left(\sum_{n=N}^{\infty}\left|\frac{\widehat{g}(n)}{\left\|\varphi_{n}(\cdot)\right\|}\right|^{2}\right)^{\frac{1}{2}}, \quad \lambda \in \Omega .
\end{aligned}
$$

Letting $N \longrightarrow \infty$, the right-hand side of (3.16) approaches zero without depending on $\lambda$, proving the uniform convergence on $\Omega$. Therefore, $f$ is analytic on compact subsets of $\mathbb{C}$, i.e., $f$ is entire. When $\varphi_{m}\left(\xi_{0}\right)=0$, for some $m \in \mathbb{N}_{0}$, the proof is similar to the one above, but in this case we should notice that $f\left(\lambda_{m}\right)=0$.

Remark 3.2. The above proof is based on the theory of differential operators. In 11 a sampling theorem is derived for integral transforms whose kernels are the resolvent kernels of the Fredholm integral operator of the second kind; see also [24]. The proof of uniform convergence of the sampling result of [1, 2] is derived using Carleman's inequality [6] p. 50]. Examples were given in [1] where the kernel of the integral operator is defined on infinite intervals, while the theory of [6] is developed for compact intervals; see [6, p. 11]. We can give a proof which does not depend on Carleman's inequality. We might use the eigenfunction expansion

$$
\Phi(x, \lambda)=\sum_{n=0}^{\infty} \frac{\varphi_{n}\left(\xi_{0}\right) \omega_{\alpha, \gamma}(\lambda)}{\left(\lambda-\lambda_{n}\right)\left\|\varphi_{n}(\cdot)\right\|^{2}} \varphi_{n}(x),
$$


which results from using the definition of $\Phi(\cdot, \lambda)$ and Green's identity. Such a proof, where the Weierstrass test can be used, covers the examples of [1, when the intervals are not finite. The other advantage offered by the theory of differential operators is that for any $\xi_{0} \in[a, \infty)$, the corresponding kernel $\Phi_{n}(\cdot, \lambda)$ is a welldefined $L^{2}$-function. This is not the case when we have resolvent kernels of square integrable symmetric kernels. Thus in [1, the square integrability of the kernels of the sampled integral transforms must be added. This makes no serious restrictions on the results of [1] since the parameter $\xi_{0}$ exists almost every where such that this condition is fulfilled.

\section{EXAMPLES}

Example 4.1. In this example we consider the Legendre equation

$$
-\left(\left(1-x^{2}\right) y^{\prime}\right)^{\prime}+\frac{1}{4} y^{\prime}=\lambda y, \quad-1<x<1 .
$$

The solutions $u, v$ of $(4.1)$ when $\lambda=0$, for which $\left(1-x^{2}\right) W_{x}(u, v) \equiv 1$ on $(-1,1)$ are $u(x)=\frac{1}{2} \tanh ^{-1} x$ and $v(x)=-1$. Both are $L^{2}(-1,1)$-functions. Hence the limitcircle case holds at the singular points \pm 1 . The boundary conditions associated with (4.1) are [8, 13]

$$
\begin{gathered}
(S y)_{1}^{-}(-1) \cos \alpha+(S y)_{2}^{-}(-1) \sin \alpha=0, \\
(S y)_{1}^{+}(1) \cos \gamma+(S y)_{2}^{+}(1) \sin \gamma=0,
\end{gathered}
$$

where

$$
(S y)_{1}^{\mp}(\mp 1)=\lim _{x \rightarrow \mp 1}\left(1-x^{2}\right) W_{x}(y, v), \quad(S y)_{2}^{\mp}(\mp 1)=-\lim _{x \rightarrow \mp 1}\left(1-x^{2}\right) W_{x}(y, u) .
$$

Let $P_{w}(\cdot)$ and $Q_{w}(\cdot)$ be the Legendre functions of the first and second type respectively, i.e.,

$$
\begin{gathered}
P_{w}(z)={ }_{2} F_{1}\left(-w, w+1 ; 1 ; \frac{1-z}{2}\right) \\
Q_{w}(z)=\frac{\sqrt{\pi} \Gamma(w+1)}{\Gamma\left(w+\frac{3}{2}\right)(2 z)^{(w+1)}}{ }_{2} F_{1}\left(\frac{1}{2} w+1, \frac{1}{2} w+\frac{1}{2} ; w+\frac{3}{2} ; z^{-2}\right)
\end{gathered}
$$

where ${ }_{2} F_{1}(a, b ; c, \cdot)$ is the well-known hypergeometric function and $\Gamma(\cdot)$ is the Gamma function; see [16]. The domain of the definition of $Q_{w}(z)$ is the set of all $z \in \mathbb{C}$ such that $|z|>1$, but it can be extended to include the interval $(-1,1)$; see [16. In the notation of Section 2 above, we have the following (cf. [13]):

$$
\begin{aligned}
& \phi(x, \lambda)=\left[\cos \alpha-\sin \alpha\left(\psi\left(\sqrt{\lambda}+\frac{1}{2}\right)+\mu\right)\right] P_{\sqrt{\lambda}-\frac{1}{2}}(-x)-\sin \alpha Q_{\sqrt{\lambda}-\frac{1}{2}}(-x), \\
& \chi(x, \lambda)=\left[-\cos \gamma-\sin \gamma\left(\psi\left(\sqrt{\lambda}+\frac{1}{2}\right)+\mu\right)\right] P_{\sqrt{\lambda}-\frac{1}{2}}(x)-\sin \gamma Q_{\sqrt{\lambda}-\frac{1}{2}}(x),
\end{aligned}
$$

$$
\omega_{\alpha, \gamma}(\lambda)=-2(\cos \alpha \cos \gamma) B(s)+\sin (\alpha-\gamma) A(s)-\frac{1}{2}(\sin \alpha \sin \gamma)\left(\frac{1-A^{2}(s)}{B(s)}\right),
$$


where $\psi(z)=\Gamma^{\prime}(z) / \Gamma(z), \mu$ is the Euler constant and

$$
A(s):=-\sin s \pi-\frac{2}{\pi}\left(\psi\left(-s+\frac{1}{2}\right)+\mu\right) \cos s \pi, \quad B(s):=-\frac{\cos s \pi}{\pi},
$$

$s:=\sqrt{\lambda}$ with a branch taken along the negative part of the real axis. Let $\xi_{0}$ be a fixed point of $(-1,1)$. Then

$$
\Phi(x, \lambda)= \begin{cases}\phi\left(\xi_{0}, \lambda\right) \chi(x, \lambda), & -1<\xi_{0} \leq x<1, \\ \phi(x, \lambda) \chi\left(\xi_{0}, \lambda\right), & -1<x \leq \xi_{0}<1 .\end{cases}
$$

Applying Theorem 2.1 of Section 2 above, the integral transform

$$
f(\lambda)=\int_{-1}^{1} \bar{g}(x) \Phi(x, \lambda) d x, \quad g(\cdot) \in L^{2}(-1,1),
$$

has the (interpolation) sampling expansion

$$
f(\lambda)=\sum_{n=0}^{\infty} f\left(\lambda_{n}\right) \frac{\omega_{\alpha, \gamma}(\lambda)}{\left(\lambda-\lambda_{n}\right) \omega_{\alpha, \gamma}^{\prime}\left(\lambda_{n}\right)},
$$

where $\left\{\lambda_{n}: n \in \mathbb{N}_{0}\right\}$ are the zeros of $\omega_{\alpha, \gamma}(\lambda)$. In the special case $\alpha=\gamma=0$, the kernel $\Phi(\cdot, \lambda)$ becomes

$$
\Phi(x, \lambda)= \begin{cases}P_{\sqrt{\lambda}-\frac{1}{2}}\left(-\xi_{0}\right) P_{\sqrt{\lambda}-\frac{1}{2}}(x), & -1<\xi_{0} \leq x<1, \\ P_{\sqrt{\lambda}-\frac{1}{2}}(-x) P_{\sqrt{\lambda}-\frac{1}{2}}\left(\xi_{0}\right), & -1<x \leq \xi_{0}<1 .\end{cases}
$$

A simple calculation yields that the sampling series of the corresponding integral transform will have the form

$$
f(\lambda)=\sum_{n=0}^{\infty} f\left(n^{2}+n+\frac{1}{4}\right) \frac{(2 n+1) \sin \pi\left(\sqrt{\lambda}-n-\frac{1}{2}\right)}{\pi\left(\lambda-n^{2}-n-\frac{1}{4}\right)} .
$$

Example 4.2. The Bessel equation

$$
-y^{\prime \prime}+\frac{\nu^{2}-\frac{1}{4}}{x^{2}} y=\lambda y, \quad 0<x \leq 1,
$$

where $0 \leq \nu \leq 1, \nu \neq \frac{1}{2}$, has the $L^{2}(0,1)$-solutions $u(x)=\frac{1}{\sqrt{2 \nu}} x^{\frac{1}{2}-\nu}, v(x)=$ $\frac{1}{\sqrt{2 \nu}} x^{\frac{1}{2}+\nu}$ when $\lambda=0$, i.e., the limit circle case holds at the left end point. Also, $W_{x}(u, v)=1,0<x \leq 1$. Let us have the following two boundary conditions:

$$
(S y)_{1}(0) \cos \alpha+(S y)_{2}(0) \sin \alpha=0, \quad y(1)=0 .
$$

In the above notations (see [11], [19])

$$
\begin{aligned}
\phi(x, \lambda) & =-\frac{\pi \sqrt{x}}{2 \sin \nu \pi}\left[J_{\nu}(s x) J_{-\nu}(s)-J_{-\nu}(s x) J_{\nu}(s)\right], \\
\chi(x, \lambda) & =\left[\sin \alpha\left(S \theta_{\lambda}\right)_{2}(0)+\cos \alpha\left(S \theta_{\lambda}\right)_{1}(0)\right] \phi(x, \lambda) \\
& -\left[\cos \alpha\left(S \phi_{\lambda}\right)_{1}(0)+\sin \alpha\left(S \phi_{\lambda}\right)_{2}(0)\right] \theta(x, \lambda),
\end{aligned}
$$


where

$$
\begin{aligned}
& \theta(x, \lambda)=\frac{s \sqrt{x}}{2 \sin \nu \pi}\left[J_{\nu}(s x) J_{-\nu}^{\prime}(s)-J_{-\nu}(s x) J_{\nu}^{\prime}(s)\right]+\frac{\phi(x, \lambda)}{2}, \\
& \left(S \phi_{\lambda}\right)_{1}(0)=\frac{\pi \sqrt{\nu}}{\sqrt{2} \sin \nu \pi}\left(\frac{s}{2}\right)^{-\nu} \frac{1}{\Gamma(1-\nu)} J_{\nu}(s), \\
& \left(S \phi_{\lambda}\right)_{2}(0)=-\frac{\pi \sqrt{\nu}}{\sqrt{2} \sin \nu \pi}\left(\frac{s}{2}\right)^{\nu} \frac{1}{\Gamma(1+\nu)} J_{-\nu(s)}, \\
& \left(S \theta_{\lambda}\right)_{1}(0)=\frac{\pi \sqrt{\nu}}{\sqrt{2} \sin \nu \pi}\left(\frac{s}{2}\right)^{-\nu} \frac{1}{\Gamma(1-\nu)}\left[s J_{\nu}^{\prime}(s)+\frac{1}{2} J_{\nu}(s)\right], \\
& \left(S \theta_{\lambda}\right)_{2}(0)=-\frac{\pi \sqrt{\nu}}{\sqrt{2} \sin \nu \pi}\left(\frac{s}{2}\right)^{\nu} \frac{1}{\Gamma(1+\nu)}\left[s J_{-\nu}^{\prime}(s)+\frac{1}{2} J_{-\nu}(s)\right]
\end{aligned}
$$

and $s=\sqrt{\lambda}$. Hence the eigenvalues of the present problem, $\left\{\lambda_{n}: n \in \mathbb{N}\right\}$ are the zeros of the function

$$
\omega_{\alpha}(\lambda)=2 \begin{cases}s^{-\nu} J_{\nu}(s) 2^{2 \nu} \frac{\Gamma(1+\nu)}{\Gamma(1-\nu)} \cot \alpha-s^{\nu} J_{-\nu}(s), & \alpha \neq 0, \\ s^{-\nu} 2^{\nu} \Gamma(1+\nu) J_{\nu}(s), & \alpha=0 .\end{cases}
$$

In a way similar to that of the previous example we could derive the sampling representation

$$
f(\lambda)=\sum_{n=0}^{\infty} f\left(\lambda_{n}\right) \frac{\omega_{\alpha, \gamma}(\lambda)}{\left(\lambda-\lambda_{n}\right) \omega_{\alpha, \gamma}^{\prime}\left(\lambda_{n}\right)}
$$

for the transform

$$
f(\lambda)=\int_{0}^{1} \bar{g}(x) \Phi(x, \lambda) d x, \quad g(\cdot) \in L^{2}(0,1),
$$

where

$$
\Phi(x, \lambda)= \begin{cases}\phi\left(\xi_{0}, \lambda\right) \chi(x, \lambda), & 0<\xi_{0} \leq x \leq 1, \\ \phi(x, \lambda) \chi\left(\xi_{0}, \lambda\right), & 0<x \leq \xi_{0} \leq 1 .\end{cases}
$$

\section{ACKNOWLEDGMENTS}

This work was carried out during the author's visit to the Department of Mathematics at Arizona State University. He is grateful to Professor Joaquin Bustoz for his hospitality and for reading the manuscript and improving its language. Also,

the author is thankful to Professor Charles Fulton for fruitful discussions.

\section{REFERENCES}

1. M.H. Annaby and M.A. El-Sayed, Kramer-type sampling theorems associated with Fredholm integral operators, Methods Appl. Anal. 2 (1995), 76-91. MR 96h:45011

2. M.H. Annaby and A.I. Zayed, On the use of Green's function in sampling theory, J. Integral Equations and Applications, 10 (1998), 117-139. MR 99g:34061

3. M.H. Annaby and G. Freiling, Sampling expansions associated with Kamke problems, Math. Z. 234 (2000), 163-189. MR 2001b:34024

4. M.H. Annaby and P.L. Butzer, On sampling associated with singular Sturm-Liouville eigenvalue problems: the limit-circle case, to appear. 
5. P.L. Butzer and G. Nasri-Roudsari, Kramer's sampling theorem in signal analysis and its role in mathematics. In: Image Processing, Mathematical Methods and Applications, The Institute of Mathematics and its Applications, New series, No 61, Clarendon Press, Oxford 1997, 49-95.

6. J.A. Cochran, The Analysis of Linear Integral Equations, McGraw-Hill, New York, 1972. MR 56:6301

7. E.A. Coddington and N. Levinson, Theory of Ordinary Differential Equations, McGraw-Hill, New York, 1955. MR 16:1022b

8. N. Dunford and J.T. Schwarz, Linear Operators, II, Wiley, New York, 1963. MR 32:6181

9. W.N. Everitt, G. Schöttler and P. L. Butzer, Sturm-Liouville boundary value problems and Lagrange interpolation series, Rend. Math. Appl. 14 (1994), 87-126. MR 95j:34040

10. W.N. Everitt, A note on the self-adjoint domains of second-order differential equations, Quart. J. Math. 13 (1963), 41-45. MR 26:1534

11. C.T. Fulton, Parametrizations of Titchmarsh's ' $m(\lambda)$ '-Function in The Limit-Circle Case, Doctoral thesis, RWTH-Aachen, 1973.

12. C.T. Fulton, Parametrizations of Titchmarsh's $m(\lambda)$-function in the limit-circle case, Trans. Amer. Math. Soc. 229 (1977), 51-63. MR 56:8950

13. C.T. Fulton, Expansions in Legendre functions, Quart. J. Math. 33 (1982), 215-222. MR 83e:34030

14. A. Haddad, K. Yao and J. Thomas, General methods for the derivation of the sampling theorems, IEEE Trans. Inf. Theory, IT-13 (1967), 227-230.

15. H.P. Kramer, A generalized sampling theorem, J. Math. Phys. 38 (1959), 68-72. MR 21:2550

16. N.N. Lebedev, Special Functions and Their Applications, Prentice Hall, Englewood Cliffs, N.J., 1965. MR 30:4988

17. M.A. Naimark, Linear Differential Operators I, Elementary theory of linear differential operators, Frederick Ungar Publishing, New York, 1967. MR 35:6885

18. I. Stakgold, Green's Functions and Boundary Value Problems, second edition, Wiley, New York, 1998. MR 99a:35002

19. E. Titchmarsh, Eigenfunction Expansions Associated with Second-Order Differential Equations Part I, Clarendon Press, Oxford, 1962. MR 31:426

20. A.I. Zayed, G. Hinsen and P.L. Butzer, On Lagrange interpolation and Kramer-type sampling theorems associated with Sturm-Liouville problems, SIAM J. Appl. Math. 50 (1990), 893-909. MR 91c:94011

21. A.I. Zayed, On Kramer's sampling theorem associated with general Sturm-Liouville problems and Lagrange interpolation, SIAM J. Appl. Math. 51 (1991), 575-604. MR 92b:34038

22. A.I. Zayed, A new role of Green's function in interpolation and sampling theory, J. Math. Anal. Appl. 175 (1993), 222-238. MR 94d:41011

23. A.I. Zayed, Advances in Shannon's Sampling Theory, CRC Press, Boca Raton, 1993. MR 95f:94008

24. A.I. Zayed, Sampling in a Hilbert space, Proc. Amer. Math. Soc. 124 (1996), 3767-3776. MR 97b:41007

Department of Mathematics, Faculty of Science, Cairo University, Giza, Egypt

E-mail address: mnaby@math-sci.cairo.eun.eg

Current address: Department of Mathematics, Arizona State University, P.O. Box 871804, Tempe, Arizona 85287-1804

E-mail address: annaby@math.la.asu.edu 